\title{
Bossy or Wimpy: Expressing Social Dominance by Combining Gaze and Linguistic Behaviors
}

\author{
Nikolaus Bee $^{1}$, Colin Pollock ${ }^{2}$, Elisabeth Andre ${ }^{1}$, and Marilyn Walker ${ }^{2}$ \\ 1 Institute of Computer Science, Augsburg University, 86135 Augsburg, Germany \\ \{andre|bee\}@informatik. uni-augsburg.de \\ 2 University of California, Santa Cruz \\ $\{$ colin|maw $\}$ @soe.ucsc.edu
}

\begin{abstract}
This paper examines the interaction of verbal and nonverbal information for conveying social dominance in intelligent virtual agents (IVAs). We expect expressing social dominance to be useful in applications related to persuasion and motivation; here we simply test whether we can affect users' perceptions of social dominance using procedurally generated conversational behavior. Our results replicate previous results showing that gaze behaviors affect dominance perceptions, as well as providing new results showing that, in our experiments, the linguistic expression of disagreeableness has a significant effect on dominance perceptions, but that extraversion does not.
\end{abstract}

Keywords: intelligent virtual agents, social dominance, verbal and nonverbal behaviors, personality, evaluation

\section{Introduction}

This paper examines the interaction of verbal and nonverbal information for conveying social dominance in intelligent virtual agents (IVAs). Our results are of immediate practical value in understanding the issues involved in designing IVAs for various applications; appropriately parametrized IVAs can communicate social dominance by head movement and gaze behaviors $[2,5]$, enthusiasm and friendliness by gesture [6], and Big Five personality traits by linguistic behaviors [11]. Therefore, we should be able to construct IVAs to communicate combinations of these different traits in order to achieve various conversational goals [1]. For example, because studies of human-human communication suggest persuasiveness is increased when the speaker is socially dominant and either extraverted or disagreeable $[3,12,7,13]$, we expect expressing social dominance will be useful in applications related to persuasion and motivation.

We draw on two distinct theories of individual differences in human traits, and the way these traits are communicated: the Big Five theory of personality $[6,17]$; and (2) Mehrabian's Pleasure, Arousal, Dominance (PAD) model, which ties personality to fundamental human emotions [14,13]. From the Big Five theory, we concentrate on the traits of extraversion and agreeableness, and from the PAD model, we focus on the dominance trait. Mehrabian (1996) reports on a meta-analysis of studies of human-human communication that suggests 
that dominance and extraversion are positively correlated, while dominance and agreeableness are negatively correlated [15]. We aim to test Mehrabian's claims with IVAs that produce utterances whose linguistic form conveys extraversion and agreeableness, while their gaze behaviors are intended to vary dominance perceptions. Our hypothesis is that when we combine verbal and nonverbal communication in a single stimulus, both will influence the participants' perceptions of the speaker's social dominance and personality traits.

To date, there have been few studies using procedurally generated conversational behavior that have examined the interaction of different modes. Rather, work has focused on providing the underlying fundamental capabilities that make such studies possible. For example, Mignault \& Chaudhuri (2003) found that a bowed head is perceived as submissive, while a raised head expresses dominance [16]. Bee and colleagues use the Alfred IVA, that we also use in our experiments (See Fig. 1), to investigate the effects on dominance of varying facial expression, and head and eye gaze direction [2]. They found that a lowered head was perceived as less dominant than a raised head, and that facial expressions defined as joy, disgust, anger and neutral all had a significantly positive influence on dominance perceptions. While these studies employed static images to measure dominance perceptions, Lance \& Marsella (2008) used animated virtual characters with a gaze model for emotional expression based on Mehrabians PAD model [9]. They found that a virtual character with either a raised head or a bowed body and/or fast movements appears more dominant. Low dominance was found for a bowed head and/or a neutral body posture without fast movements. Fukayama and colleagues (2002) propose a gaze behavior model for virtual characters based on amount and mean duration of gaze and averted gaze orientation [5]. They evaluated their model by displaying animated eyes to the users and found that eye gaze directed to the user is perceived as more dominant than eye gaze directed downwards.

On the verbal side, relevant previous work describes linguistic generators that convey Big Five Personality traits or which are intended to induce social compliance. Roque \& Traum (2007) implemented styles of conversation related to social compliance, but have not yet tested user perceptions [18]. Mairesse \& Walker show that human subjects perceive utterances as conveying the different levels of extraversion that the PERSONAGE generator was targeting; subjects also correctly perceive the level of agreeableness that the system intended $[11,10]$. Here we will use these prior results to examine the prediction that extraversion and agreeableness will affect dominance perceptions. Section 2 explains how we generate videos of recommendations combining gaze and linguistic behaviors. Section 3 describes our main experiment examining the interaction of personality and dominance. We conclude in Section 4.

\section{Generating Verbal and Nonverbal Behaviors}

We will test human perceptions of an IVA speaking restaurant recommendations generated by PERSONAGE that were reliably shown to affect the perception of 
the agreeableness and extraversion traits. We also use the Alfred IVA with facial and gaze behaviors shown to affect perceptions of social dominance $[2,5]$.

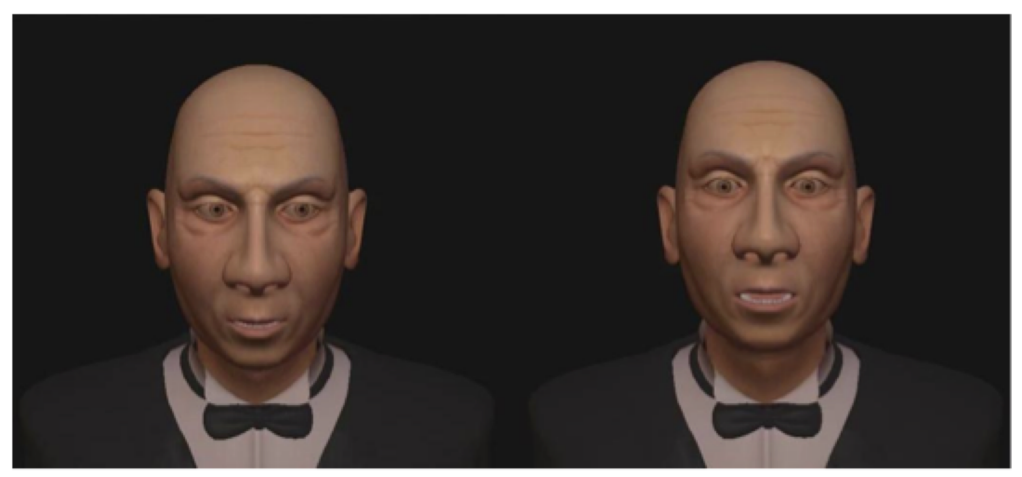

Fig. 1: Image of Low Dominance Alfred (left) and High Dominance Alfred (right)

Our generation engine for nonverbal behaviors was Alfred, a butler-like virtual character [2]. Alfred interfaces with the Microsoft Speech API in order to synchronize the audio output with the lip movements. We used the Facial Action Coding System (FACS) to control lip movements (e.g. funneling, tightening, stretching). Alfreds eyes and head are controlled with an inverse kinematics component integrated into the Horde3D GameEngine. This allows us to control each eye and head separately.

In order to target the nonverbal channel, we start from the gaze model developed by Fukayama and colleagues (2002) to specify a number of gaze parameters that influence the impression a character conveys. Their model includes two states: looking at the user and looking away from the user. Three parameters define frequency, duration (500 to $2000 \mathrm{~ms}$ ), and location of gaze. The gaze targets consist of a set of random points from either all over the scene, above, below or close to the user. The probabilities of changing state or staying in the same state depend on the amount and the mean duration of the gaze parameters. Fukayama and colleagues found that a medium amount of gaze and a mean duration between 500 to $1000 \mathrm{~ms}$ conveys a friendly gaze behavior. The orientation of the gaze direction did not play a decisive role in distinguishing between friendly and dominant gaze behavior, except that a downward gaze was considered as less dominant. While their experiments only display eyes to the user, we evaluate their model with a full virtual head that in moves his head and eyes, but set the parameters according to their findings for dominant and friendly eye gaze behavior. The submissive gaze behavior was set to a mean duration of $500 \mathrm{~ms}$, randomly changing between gazing at the user and averting the gaze. Further, we bowed Alfreds head according to the findings of $[9,16,2]$. In order to model the dominant eye gaze behavior, Alfred stares at the user when speaking the restaurant recommendation. We used Alfred's neutral facial expression [2]. 
Table 1: Example utterances generated by Personage to be spoken by Alfred.

\begin{tabular}{|c|c|c|}
\hline Trait & Level & Recommendation \\
\hline Extraversion & Low & I mean, Amy's Bread isn't as bad as the others. \\
\hline Extraversion & High & $\begin{array}{l}\text { I am sure you would like Bond Street, you know. } \\
\text { Basically, the food is great and the atmosphere is } \\
\text { good, wth friendly service. }\end{array}$ \\
\hline Agreeableness & Low & $\begin{array}{l}\text { Basically, everbody knows that John's Pizzeria is the } \\
\text { only restaurant that is any good. Tis eatin gplace } \\
\text { offers like, mediocre ambience, even if it's bloody } \\
\text { expensive. Actually, the waiters aren't good. }\end{array}$ \\
\hline Agreeableness & High & $\begin{array}{l}\text { You want to know more about John's Pizzeria? Oh } \\
\text { well, it's price is around } 20 \text { dollars, so it's one of my } \\
\text { favorite eating houses, and it offers kind of dainty } \\
\text { food, you know, you see? }\end{array}$ \\
\hline
\end{tabular}

For verbal behaviors, we use the Personage generator of Mairesse and Walker, along with parameter models that were shown in their work to generate utterances reliably perceived as either highly introverted or extraverted, or highly disagreeable or agreeable $[10,11]$. Personage is based on an extended set of modules from the standard NLG architecture, with modules for content planning, sentence planning, and surface realization. In contrast with many generators, PERSONAGE provides many parameters to support pragmatic transformations that affect human perceptions of the speakers personality traits. Examples of the eight utterances generated by PERSONAGE used in our experiment are in Table 1. Four utterances target extraversion and four target agreeableness, with two utterances at the low end of the scale (disagreeable, introverted) and two at the the high (agreeable, extraverted) end. In a pretest, we confirmed that voice realization of these utterances using Loquendo's Simon voice maintained previous findings on the perception of these utterances.

\section{Experimental Study}

Our main goal is to understand how people form impressions of an IVA's social dominance when its verbal and nonverbal communication are observed simultaneously. The simplest prediction is (H1). In addition, because of Mehrabian's findings, we predict (H2) linguistic extraversion and agreeableness will affect dominance ratings, and that gaze-based dominance will impact personality trait ratings.

- (H1) Personality traits and gaze will both effect the perception of dominance, extraversion and agreeableness.

- (H2) Extraversion will increase the perception of dominance, while agreeableness will decrease it. Gaze-based dominance will increase the perception of extraversion.

Method. Nineteen subjects participated in the experiment through a website. Nine were male and ten were female with a mean age of 26.5 years. English was the first language of all participants. Subjects first read an instruction page that described their task, but provided no information about the domain. Then 
the experimental stimuli were presented one at a time, in random order. For the verbal content, we used eight restaurant recommendations targeting introversion (low extraversion), extraversion, disagreeableness (low agreeableness), and agreeableness, as illustrated in Table 1. Each recommendation was presented as male speech generated using the Loquendo TTS voice Simon, and combined with both dominant and submissive gaze behaviors. For each stimulus, the participants answered eleven questions about their impressions of the speaker's personality and the naturalness of the utterance: four questions for extraversion and agreeableness were from the Ten Item Personality Inventory (TIPI) [8], and six questions were from Mehrabian's dominance scale [14]. Given the large number of judgements required for all stimuli, and the fact that stimuli may be viewed repeatedly, we use a within-subjects design.

Results. In order to test whether personality traits and gaze factors both affect the perception of dominance, extraversion and agreeableness, we first establish that each of the three factors (gaze, linguistic extraversion, and linguistic agreeableness) significantly affects each of the three dependent variables (ratings of dominance, extraversion, and agreeableness), confirming H1. See Fig. 2. One way ANOVAs for each factor show significant differences for the effect of linguistic extraversion on perception of extraversion $(\mathrm{F}(1,151)=6.68, \mathrm{p}=.01)$, linguistic agreeableness on the perception of agreeableness $(\mathrm{F}(1,151)=71.91, \mathrm{p}$ $<.001)$ and gaze-based dominance on the perception of dominance $(\mathrm{F}(1,303)=$ $6.36, \mathrm{p}=.01)$.
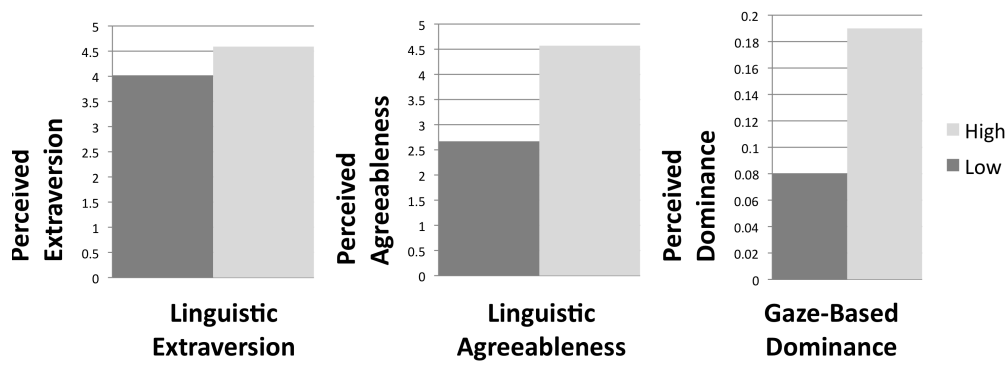

Fig. 2: Perception of Verbal and Non-verbal Cues

We then turned to the cross-modal effects on perception. See Fig. 3. Results indicate that linguistic agreeableness inversely affects the perception of dominance $(\mathrm{F}(1,151)=18.54, \mathrm{p}<.001)$, and that gaze-based dominance affects the perception of extraversion $(\mathrm{F}(1,303)=13.7, \mathrm{p}<.001)$, confirming H2. See Fig. 3. To examine interaction effects, we ran a two-way ANOVA testing for the effect of linguistic extraversion and gaze-based dominance on the perception of extraversion. This showed a significant result for an interaction $(\mathrm{F}(3,151)=4.37, \mathrm{p}=$ .04). See the right hand side of Fig. 3 which illustrates that when linguistic extraversion is high, gaze behavior has no effect on dominance, thus disconfirming one aspect of $\mathrm{H} 2$. 

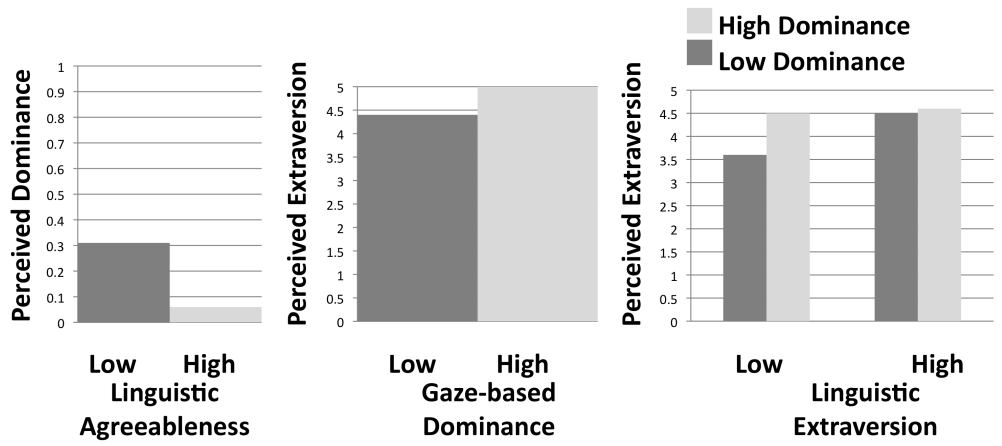

Fig. 3: Perceived Dominance and Extraversion

\section{Discussion and Conclusion}

Our studies suggest that we succeeded in conveying linguistic personality traits and visual dominance. While our results may be specific to our stimuli, all stimuli were generated procedurally using parameters that can be tested in future work. Our results show that perceptions of agreeableness and extraversion were consistent with the intended perceptual target, and that more dominant faces were perceived as such. Our novel result is the demonstration that linguistic personality traits influence the perception of dominance and that gaze-based dominance influences the perception of personality traits. Other such perceptual interactions could be investigated in future work. In future work we aim to test whether conveying dominance affects persuasiveness or motivation as predicted by previous work $[3,12]$.

An unexpected and interesting finding is that our results suggest that neither the verbal or nonverbal channel is dominant; rather, when cues to dominance are inconsistent, perception depends on the trait being conveyed. For example, the character's gaze and head pose communicated dominance independently of the expression of linguistic extraversion, but in the case of linguistic agreeableness, the linguistic modality had a greater effect than the visual modality. Thus while previous work suggests that the nonverbal channel carries $60 \%$ of the meaning in conversation [14], our results support the findings of Ekman and colleagues (1980), who show that channel dominance depends on what is being communicated; in particular that affective, personality, and informational aspects of meaning are independently affected by different channel components in different situations [4].

\section{Acknowledgements}

The work reported in this paper is partially supported by DynaLearn, co-funded by the EU within the 7th Framework Programme, Project no. 231526, and by award 20101185 from NPS to UCSC on Perlocutions and Dialog. 


\section{References}

1. André, E., Rist, T., van Mulken, S., Klesen, M., Baldes, S.: The automated design of believable dialogues for animated presentation teams. Embodied conversational agents pp. 220-255 (2000)

2. Bee, N., Franke, S., André, E.: Relations between facial display, eye gaze and head tilt: Dominance perception variations of virtual agents. In: Affective Computing and Intelligent Interaction (2009)

3. Cialdini, R.: Influence: The psychology of persuasion. Quill New York (1993)

4. Ekman, P., Friesen, W., O'Sullivan, M., Scherer, K.: Relative importance of face, body, and speech in judgments of personality and affect. Journal of Personality and Social Psychology 38(2), 270-277 (1980)

5. Fukayama, A., Ohno, T., Mukawa, N., Sawak, M., Hagita, N.: Messages embedded in gaze of interface agents - impression management with agent's gaze. In: CHI '02: Proc. of the SIGCHI conference on human factors in computing systems. pp. 41-48 (2002)

6. Funder, D.C.: The Personality Puzzle. W. W. Norton \& Company, New York, 2nd edn. (1997)

7. Furnham, A.: Language and personality. In: Giles, H., Robinson, W. (eds.) Handbook of Language and Social Psychology. Winley (1990)

8. Gosling, S.D., Rentfrow, P.J., Swann, W.B.: A very brief measure of the big five personality domains. Journal of Research in Personality 37, 504-528 (2003)

9. Lance, B., Marsella, S.: The relation between gaze behavior and the attribution of emotion: An empirical study. In: Intelligent Virtual Agents. pp. 1-14. Springer (2008)

10. Mairesse, F., Walker, M.A.: PERSONAGE: Personality generation for dialogue. In: Proceedings of the 45th Annual Meeting of the Association for Computational Linguistics (ACL). pp. 496-503 (2007)

11. Mairesse, F., Walker, M.A.: Trainable generation of Big-Five personality styles through data-driven parameter estimation. In: Proceedings of the 46th Annual Meeting of the Association for Computational Linguistics (ACL) (2008)

12. Marwell, G., Schmitt, D.: Dimensions of compliance-gaining behavior: An empirical analysis. Sociometry 30(4), 350-364 (1967)

13. Mehrabian, A.: Significance of posture and position in the communication of attitude and status relationships. Psychological Bulletin 71(5), 359-372 (1969)

14. Mehrabian, A.: Framework for a comprehensive description and measurement of emotional states. Genetic Social and General Psychology Monographs 121(3), 339361 (1995)

15. Mehrabian, A.: Analysis of the big-five personality factors in terms of the PAD temperament model. Australian Journal of Psychology 48(2), 86-92 (1996)

16. Mignault, A., Chaudhuri, A.: The many faces of a neutral face: Head tilt and perception of dominance and emotion. Journal of Nonverbal Behavior 27(2), 111132 (2003)

17. Norman, W.T.: Toward an adequate taxonomy of personality attributes: Replicated factor structure in peer nomination personality rating. Journal of Abnormal and Social Psychology 66, 574-583 (1963)

18. Roque, A., Traum, D.: A model of compliance and emotion for potentially adversarial dialogue agents. In: Proceedings of the 8th SIGdial Workshop on Discourse and Dialogue (2007) 